\title{
Minireview
}

\section{The discovery of Rubisco activase - yet another story of serendipity}

\author{
Archie R. Portis $\mathrm{Jr}^{1, *} \&$ Michael E. Salvucci ${ }^{2}$ \\ ${ }^{1}$ Photosynthesis Research Unit, Agricultural Research Service, United States Department of Agriculture, Urbana, \\ IL 61801, USA ${ }^{2}$ Western Cotton Research Laboratory, Agricultural Research Service, United States Department \\ of Agriculture, 4135 East Broadway Road, Phoenix, AZ 85040, USA; *Author for correspondence (e-mail: \\ arportis@uiuc.edu; fax:+1-217-244-4419)
}

Received 4 July 2001; accepted in revised form 24 October 2001

Key words: Arabidopsis, Calvin-Benson cycle, light regulation, William Ogren, Archie Portis, Simon Robinson, Rubisco, Michael Salvucci, Christopher Somerville, 2-carboxyarabinitol 1-phosphate

\begin{abstract}
A brief history of Rubisco (ribulose bisphosphate carboxylase oxygenase) research and the events leading to the discovery and initial characterization of Rubisco activase are described. Key to the discovery was the chance isolation of a novel Arabidopsis photosynthesis mutant. The characteristics of the mutant suggested that activation of Rubisco was not a spontaneous process in vivo, but involved a heritable factor. The search for the putative factor by $2 \mathrm{D}$ electrophoresis identified two polypeptides, genetically linked to Rubisco activation, that were missing in chloroplasts from the mutant. An assay for the activity of these polypeptides, which were given the name Rubisco activase, was developed after realizing the importance of including ribulose bisphosphate (RuBP) in the assay. The requirement for ATP and the subsequent identification of activase as an ATPase came about fortuitously, the result of a RuBP preparation that was contaminated with adenine nucleotides. Finally, the ability of activase to relieve inhibition of the endogenous Rubisco inhibitor, 2-carboxyarabinitol 1-phosphate, provided an early indication of the mechanism by which activase regulates Rubisco.
\end{abstract}

Abbreviations: CA1P - 2-carboxyarabinitol 1-phosphate; FBP - fructose bisphosphate; RuBP - ribulose bisphosphate; Rubisco - ribulose bisphosphate carboxylase oxygenase; Ru5P - ribulose 5-phosphate; SBP - sedoheptulose bisphosphate

'Much discussion, both inside and outside the lecture halls, centered upon the potentially important reports of endogenous compounds which inhibit or activate RuBPCO. The debate was fiercest over reports by M. Salvucci, A. Portis and co-workers of the presence in some tissues of a protein which is involved in the activation of RuBPCO. The suggestion that the protein is an enzyme, hence the name RuBPCO activase, appeared to raise some hackles.'

J. A. M. Holtum and E. Latzko, 1987

\section{Pre-activase history - a complex and schizophrenic Rubisco}

A brief look at some of the history of Rubisco research reveals a saga of surprises that will set an appropriate stage for describing the discovery of Rubisco activase. This most abundant plant protein masqueraded for many years as 'Fraction one' protein (Wildman and Bonner 1947; Kawashima and Wildman 1970) before its carboxylation activity was uncovered, beginning in 1954 (for historical accounts, see Weissbach and Horecker 1989; A.A. Benson, this issue; and S. Wildman, this issue). Many years later, its bifunctionality was revealed by discovery of its oxygenase activity (Bowes et al. 1971). The oxygenase 
activity was an elegant explanation for both the long sought source of the substrate for photorespiration and the effects of oxygen on photosynthesis, but it engendered some heated debates rather than acclaim for the breakthrough it represented. Rubisco oxygenation went against earlier evidence for other sources of glycolate, and some expert biochemists found it difficult to accept that an oxygenase could function without a transition metal cofactor, such as iron or copper. The amount of copper associated with the protein and whether the carboxylase and oxygenase activities were really catalyzed by the same protein or could be separated from each other consumed much research effort for a time.

Even measuring the activity of Rubisco properly proved to be difficult. Many papers on measuring its kinetic properties reported various effectors and allosteric interactions. Also, it soon became apparent that even under the best of circumstances the activity of the isolated protein was not adequate to account for the measured rates of photosynthesis, unless one resorted to rapidly assaying it with lysed chloroplasts (Bahr and Jensen 1974). The significance of the early evidence of 'activation' by inorganic carbon and $\mathrm{Mg}^{2+}$ by Pon et al. (1963) was largely overlooked until 1976, when George Lorimer and colleagues clearly demonstrated that $\mathrm{CO}_{2}$ was not only a substrate, but also an activator (Lorimer et al. 1976; Lorimer 1979). This realization rendered most kinetic and regulatory studies reported before prior to 1976 largely useless. Their mechanism for a spontaneous ${ }^{1}$ carbamylation of the enzyme by $\mathrm{CO}_{2}$ followed by $\mathrm{Mg}^{2+}$ binding, which was favored at alkaline $\mathrm{pH}$, had the added benefit of providing a ready explanation for 'light activation' of the enzyme (Walker 1973) and the subsequent finding that the enzyme was modulated by light in vivo (Machler and Nosberger 1980; Perchorowicz et al. 1981). Effectors, sugar phosphates that could stimulate or inhibit Rubisco when preincubated with the enzyme, $\mathrm{CO}_{2}$, and $\mathrm{Mg}^{2+}$ before assay, then became of major interest. One of them, 2-carboxyarabinitol 1phosphate (CA1P), would come to rival activase for importance. Meanwhile, the other substrate, RuBP, received little notice even though the early paper by Pon et al. (1963) also showed that preincubation of the enzyme with RuBP led to reduced rates of activity. Later, a detailed analysis by Laing and Christeller (1976) provided evidence that RuBP was an inhibitor of activation, allowing them to extend the carbamylation model of Lorimer et al. (1976). Finally, a paper by Jordan and Chollet (1983) most clearly showed that
RuBP binding to the uncarbamylated enzyme resulted in a potent inhibition of the carbamylation/activation process.

Rubisco's many deficiencies are often mentioned in the literature, but here we will conclude this brief introduction by pointing out that Rubisco is somewhat schizophrenic-not only is it bifunctional with its oxygenase activity undoing a considerable amount of carboxylation, but one substrate, $\mathrm{CO}_{2}$, is also an essential activator, while the other, RuBP, is also an inhibitor of the very same activation process.

\section{A sickly mutant}

The key to the eventual discovery of activase was the Arabidopsis rca mutant, which was reported by Somerville et al. (1982). This mutant came through the high $\mathrm{CO}_{2}$ /low $\mathrm{CO}_{2}$ screen developed by Chris Somerville, while a postdoc in Bill Ogren's USDA laboratory located in the Agronomy Department in Turner Hall at the University of Illinois (Figure 1). The screen was designed to isolate mutants with defects in the photorespiratory pathway, a radical approach at the time for resolving controversies about metabolic pathways in plants. Mutant CS207 was just one of many that passed the initial screen, but initially it did not grow very well even under high $\mathrm{CO}_{2}$ and could easily have been discarded. Fortunately, Chris was meticulous and he took the time to backcross such plants to the wild-type in order to eliminate spurious mutations.

Chris Somerville's usual approach for characterizing these mutants was to label them with ${ }^{14} \mathrm{C}$ and then apply a simple batch ionic exchange separation in order to identify the potential lesion by the compound that accumulated. Mutant CS207 was interesting because under low $\mathrm{CO}_{2}$ it accumulated label in the strong acids, usually Calvin-Benson cycle intermediates, rather than the weak acids or bases, which were expected with mutants in the photorespiratory pathway. Archie Portis, newly arrived in Urbana himself and located in a lab directly across from Bill Ogren's, had set up an ion-exchange high pressure liquid chromatography (HPLC) system specifically to resolve Calvin-Benson cycle intermediates. Chris came to him one day with some samples and asked if he would chromatograph them for him. They were surprised to see the large labeling of the RuBP pool, suggesting that Rubisco activity was the problem. However, when Chris isolated the Rubisco protein from the mutant and assayed it, Rubisco appeared perfectly normal. 


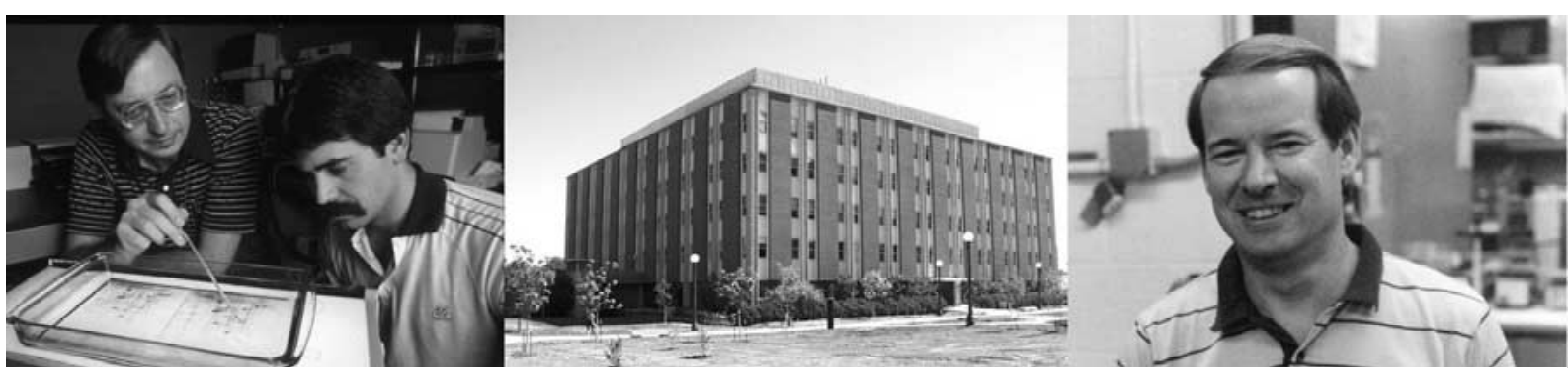

Figure 1. Left: William (Bill) Ogren $(l)$ and Michael (Mike) Salvucci $(r)$ examining 2-D gels of chloroplast extracts from wild type and rca mutant Arabidopsis. Middle: Turner Hall, University of Illinois, Urbana, Illinois, where the rca mutant was recovered and activase was discovered in the second floor labs of Ogren and Portis. Right: Archie Portis in his lab a few years after the discovery.

The emerging properties of the mutant prompted much discussion, which included Marty Spalding, another postdoc in Bill's lab at the time. It was soon realized that the methods and ideas put forth in a newly published paper by Perchorowicz et al. (1981) on Rubisco activation in wheat seedlings could be revealing. Chris quickly adapted the methods to Arabidopsis and was then able to show that the problem with the mutant was not Rubisco activity per se, but its level of activation. Thus the CS207 mutant was named $r c a$ (ribulosebisphosphate carboxylase activation). Meanwhile, in writing up the results, the mutant prompted much discussion about how this could be. If stromal $\mathrm{pH}$ or $\mathrm{Mg}^{2+}$ were a problem, work in several labs, particularly in Hans Heldt's lab by Archie and others had shown that the FBPase and SBPase activities would also be affected. Much discussion also focused on Ru5P kinase. Could a regulatory disruption of this enzyme raise the stromal RuBP to inhibitory levels for Rubisco? We suspected that the mutant was the key to understanding Rubisco regulation, but just how to proceed was not very clear.

\section{Protein gels with a missing band}

Chris Somerville soon left Bill Ogren's lab with a host of ideas that he felt could be approached by mutagenesis of Arabidopsis and the $r c a$ mutant just sat in Bill's Arabidopsis collection for some time. Then Mike Salvucci arrived in Bill's lab with an interest in isolating/characterizing photosynthesis mutants from a collection of Chlamydomonas mutants produced by Bob Spreitzer, who had just completed a postdoc in Bill's lab. Mike and Archie hit it off right from the start and soon became good friends. The first mutant Mike identified had a defect in Ru5P kinase. One day the Arabidopsis rca mutant came up in conversation, particularly the hypothesis that it might have a defect in Ru5P kinase. Mike was aware of the $r c a$ mutant from a preprint of the original manuscript that he had received in a recruitment package from Bill (on this preprint Bill wrote: 'Pursuing this mutant might also be a possibility'). Mike agreed to grow some of the $r c a$ mutant and to include an extract in one of the Ru5P kinase assays. These first experiments were not very encouraging, as the Ru5P kinase activity appeared to be normal. Further discussions ensued, leading to the hypothesis that Rubisco activation was somehow catalyzed in vivo by a protein that was missing or defective in the mutant. We mentioned this idea to Bill, who reminded us of previous reports of a Rubisco 'light activation factor,' disparagingly known among some photosynthetic carbon researchers at the time as the LAF factor, and suggested that, if we find such a protein, we should give it a name less subject to ridicule. As a result, we began calling our hypothetical protein Rubisco activase. Later on, the implication that activase was an enzyme would not be well received (Holtum and Latzko 1987). However the proliferation of reports of proteins like activase whose main function is to catalyze changes in noncovalent bonding is causing a re-assessment of how we classify and catalog enzymes (Purich 2001).

The more we discussed the 'Rubisco activase hypothesis,' the more we liked the idea and were convinced of the potential importance of the mutant in proving it. Our ideas were bolstered by recent reports from Perchorowicz and Jensen (1983) and also Weiss $(1981 \mathrm{a}, \mathrm{b})$, which where not consistent with the idea that increases in stromal $\mathrm{pH}$ and $\mathrm{Mg}^{2+}$ were solely responsible for light activation of Rubisco. Consequently, we decided to run a few gels on extracts of the mutant and wild-type - 'just to have a look' - but knowing full well that the chances of a protein actually being absent were not great. We reasoned that the pro- 
tein must be fairly small, because it would have to be fairly abundant to activate all of the Rubisco, yet appear relatively faint on gels to have escaped detection by John Ellis and others who had already identified many of the chloroplast stromal proteins. When we analyzed the gels we saw no differences at the low molecular weight end, but one of us (AP) spotted a difference at about $42 \mathrm{kDa}$. But was the difference real and related to the mutant phenotype? Apprehensive that Bill Ogren would think that we were wasting our time, we finally showed the gels to him. If he was as excited as us was hard to discern, but his immediate comment was to suggest that Mike make some crosses to determine if the missing band, which was quite faint in the wild type, could be separated from the $r c a$ phenotype, a high $\mathrm{CO}_{2}$ requirement for growth. While waiting for the crosses, we decided to isolate chloroplasts from the mutant and wild type and run 2D gels on the stromal extracts. Unfortunately, obtaining chloroplasts from Arabidopsis first required the isolation of protoplasts, which in itself was rather difficult and time consuming. But the effort was rewarded by a clear result: the gels showed that not one but two polypeptides were missing from mutant chloroplasts, verifying that a protein with possibly two subunits was indeed missing in the mutant and that the protein was chloroplastic.

The results of the crosses were also positive and we soon had both genetic and physiological evidence that a previously unknown protein was required to maintain Rubisco in a highly active state at normal $\mathrm{CO}_{2}$ concentrations. The next step was obviously to purify and characterize this new protein, but we had a major problem: how to assay its activity so that it could be followed during purification? Since reports in the literature indicated that the light activation of Rubisco could be observed in isolated chloroplasts (e.g. Heldt et al. 1978), Archie Portis began a long series of experiments to characterize this process in more detail and to see if a light dependent increase in Rubisco activity could be observed following lysis of intact chloroplasts. After almost a year of experiments some progress was being made in showing that light activation in chloroplasts clearly involved more than stromal $\mathrm{pH}$ and $\mathrm{Mg}^{2+}$ changes, but otherwise things were looking rather grim as all attempts at showing activation after lysis of the chloroplasts had failed. Meanwhile, Mike Salvucci had accepted a faculty position at a university in the northeast and left Urbana in December 1984, with best-laid plans to collaborate with Bill and Archie on the activase problem.

\section{My kingdom for an assay!}

Extremely frustrated at the lack of a way to identify and follow this new protein in the wild-type plants, we had to consider publishing our findings as they were and mulled things over during the winter holidays. Archie Portis went to the Eighth Annual Symposium in Plant Physiology at UC Riverside in January 1985 and presented some unrelated work on chloroplast metabolism. Metabolite regulation of chloroplast carbon metabolism was a major theme, as fructose 2,6-bisphosphate was just recently reported. While listening to one of the talks, he realized that all of the previous experiments with lysed chloroplast had never included any RuBP in the incubation reaction mixture-it had only been present in the assay mixtures. Given our knowledge of Jordan's experiments on the effects of RuBP on spontaneous activation (Jordan and Chollet 1983) and some rather curious results reported when RuBP was present in chloroplast lysates (Lendzian 1978; Sicher et al. 1981), this was quite an oversight. Immediately upon returning to the lab, a rather simple experiment was conducted. Intact chloroplasts were lysed into a solution containing RuBP and an electron transport acceptor, and Rubisco activity was then followed for the next few minutes in either light or darkness. The results were quite exciting as the initial activity increased with time in light while it decreased in darkness, such that, after 6 min a fourfold difference was observed.

\section{Who needs a real job when you can be chasing activase?}

Unknown to Archie Portis, Mike Salvucci had called Bill Ogren asking if he could return to his postdoc position, because key promises made to him in his new faculty position were not being fulfilled. Bill told him 'no problem, your bench is still open,' but in reality he had to pull a few governmental strings, as this was an extraordinary request. Immediately upon his return in February 1985, Archie showed Mike and Bill the data, which indicated that we now had a possible assay for this new protein. We then set about isolating chloroplasts from the wild-type and mutant Arabidopsis plants in order to use them in the lysed chloroplast assay and as a source of stromal extract for a reconstituted assay. Again, the effort was rewarded by a clear result: the light-dependent increase in Rubisco activity only occurred with wild type extracts. The 
final piece was in hand - an in vitro assay that showed that the missing protein was required for the increase in Rubisco activity.

\section{Publication frustration and paranoia}

With a few clean-up experiments to follow, we now felt in a position to publish a 'landmark' paper in a prestigious journal. First we tried Nature, but the paper was rejected without being sent out for review. Next we tried Science, and the reviewers raised many questions, but we had an opportunity to respond to the criticisms. One reviewer asked how we could be certain the missing band was really associated with the Rubisco activity increase when we had not yet purified it. We went back and forth with the reviewers over this issue, but our genetic evidence linking lack of activity with absent proteins was of little consequence to the reviewers and editor. It seemed that combining genetics, physiology, and biochemistry counted for little in trying to overthrow the existing dogma that activation of Rubisco was a spontaneous process in vivo as well as in vitro.

At this point, we had become extremely frustrated, and paranoia about getting published in 1985 began to set in. The paper was submitted in April and by October it had not been accepted or rejected, but was still out for review. Also preliminary reports were appearing about another process affecting Rubisco activation. Furthermore, the activation assay was so easy to perform anyone might have also discovered it, and we were well on our way to purifying the activase protein. In the course of conversation, Bill mentioned the problems we were having with publication to Govindjee, also at Urbana. Govindjee suggested that we submit the manuscript as a short communication to Photosynthesis Research, which he conveniently edited. Consequently, Mike dashed off a letter pulling the paper from further consideration by Science, and we submitted the manuscript to Photosynthesis Research. The manuscript (Salvucci et al. 1985) was immediately accepted by Govindjee and printed without typesetting in the November 1985 issue.

\section{A fortunate case of contamination}

In earlier days, biochemists were always worried about the purity of their reagents and enzymes because many had to be synthesized or isolated in-house; com- mercial sources were unavailable. Experiments that worked one day, but not the next, were usually followed up by a systematic investigation. With today's emphasis on prepackaged kits and the availability of 'ultra-pure' reagents not much thought is given to purity anymore. Thus, if an experiment suddenly does not work, one is likely to conclude that the researcher must just 'have messed up.' Also there seems to be some hesitation about even doing an experiment if a critical component is not readily available. This change is interesting, because there are numerous instances of important advances resulting from a contaminated reagent or enzyme. Activase is one such case.

With an assay in hand, we now set about to purify this new protein and to conduct experiments to determine the important features of the activation mechanism. One day during the course of these experiments, we did not have activation activity in any of the controls. The experiment was repeated the next day with similar results. With some panic beginning to set in, we realized that a new batch of RuBP had been used for these experiments. Due to its great expense, we like most 'Rubiscologists' synthesize our RuBP from less expensive precursors. We anxiously did another experiment in which both the new and an old RuBP preparation were used and we were quite relieved to see that the old RuBP preparation still worked. Looking over how these were prepared, some additional steps had been introduced to 'improve' the synthesis protocol. In the synthesis, stoichiometric amounts of ATP were used to phosphorylate ribulose 5-phosphate. The ATP/ADP was then removed by treating the reaction with charcoal, once in the old procedure, several times in the new procedure. A quick UV spectrum of both RuBP samples revealed that the older preparation was severely contaminated with adenine nucleotide whereas the most recent one was not. Further experiments by Kit Streusand, a postdoc in Portis's lab, quickly revealed that not only was ATP required, but also the presence of thylakoids was no longer absolutely necessary (Streusand and Portis 1987). Apparently they were only acting as an ATP regenerating system.

\section{CA1P - a red herring?}

A few years before the discovery of activase, several reports appeared showing that Rubisco was inhibited in the dark (reviewed in Seemann et al. 1990). A flurry of activity ensued as researchers in at least 
five different labs raced to identify the long sought after compound responsible for light activation of Rubisco. Meanwhile, our results with activase were convincing us that Rubisco activase was the universal regulator of Rubisco and not this compound. Our confidence was bolstered by reports showing that the so-called dark inhibitor was not in all plant species and our own experiments indicating that this compound was not in Arabidopsis. Also, it was difficult to explain how a compound present mainly in the dark could prevent RuBP from inhibiting Rubisco in light. Consequently, when the inhibitor was identified as 2-carboxyarabinitol 1-phosphate (CA1P) by two different labs, both of which relied on John Pierce at Dupont for their NMR (Gutteridge et al. 1986; Berry et al. 1987), we took great pains to distance ourselves from all attempts to connect activase and CA1P.

In December of 1985, Mike Salvucci left Urbana for a position with the USDA at the University of Kentucky. Because the job was in the Tobacco and Forage Research Unit, and tobacco contained CA1P, he began investigating the relative contributions of activase and CA1P to the regulation of Rubisco in tobacco. These studies led to a collaboration with George Bowes and Gabe Holbrook, then a postdoc with George, that culminated in the identification of CA1P phosphatase, a specific phosphatase that metabolizes CA1P in light (Holbrook et al. 1989). Back in Urbana, Simon Robinson joined Archie Portis on a sabbatical from Australia and was proceeding through a detailed characterization of activase, including elucidation of its ATPase activity (Robinson and Portis 1989). Because of his extremely well organized approach, almost every experiment worked for Simon, and he produced five papers during his nine-month visit. However, a reviewer of one of the papers was not impressed, criticizing us for trying to publish an MPU (minimum publishable unit). There is another interesting anecdote from Simon's time in Urbana. One day Simon related to Archie that several years earlier he had detected a curious stromal ATPase activity during his work on the envelope transport ATPases. He had never published anything, since he could not figure out what the protein was doing but now realized that it must have been activase. Sometimes inexplicable observations eventually make sense in the end.

Simon Robinson was curious about a possible relationship between CA1P and activase, and he suggested to Archie that they get a sample of CA1P to examine its effects on Rubisco in the presence of the activase. Archie agreed and Simon contacted Joe Berry, who kindly provided a sample for the experiments. Previous work on CA1P had shown that its release from Rubisco was very slow (Berry et al. 1987) and that CA1Pase could only hydrolyze CA1P once it was released (Salvucci et al. 1988). Robinson and Portis showed that activase was indeed active towards CA1P, facilitating its release from Rubisco to make it available for metabolism by CA1P phosphatase (Robinson and Portis 1988). More importantly, Simon's experiments with CA1P laid the groundwork for later studies in Archie's lab, which established that facilitating the release of sugar phosphates that otherwise prevent carbamylation and/or catalysis was a key component in the mechanism of activase.

\section{Conclusions}

Looking back over this course of events, a number of seemingly unrelated factors combined to contribute to the discovery of activase, not the least of which was dumb luck. For example, renewed effort on the rca mutant began with the chance discovery of an Ru5P kinase mutant in Chlamydomonas. Later, the initial experiments using lysed chloroplasts and RuBP only worked because micromolar amounts of ATP/ADP had been fortuitously introduced along with the millimolar concentrations of RuBP that were being used. Also, we were fortunate that the rca mutation eliminated activase expression and that activase was abundant enough and of a size not to be obscured by other proteins on the gels. Finally, timely reports by other investigators were of paramount importance in developing a hypothesis to account for the behavior of Rubisco in the Arabidopsis rca mutant.

Undoubtedly, the most important single factor in the discovery of activase was the existence of the rca mutant of Arabidopsis. If this mutant had not been isolated and properly characterized, activase would probably have remained unknown for some time. However, it is likely that recent advances in our understanding of the conformational changes that accompany sugar phosphate binding (Duff et al. 2000) derived from the high resolution crystal structures of Rubisco (Schreuder et al. 1993) would have eventually led to the conclusion that conformational changes in Rubisco must be facilitated in some way. Also, the development of micro-sequencing techniques and expressed sequence tags would have identified activase as a chloroplast-localized ATPase, and its function would have eventually been identified through muta- 
genesis or antisense technology. However, these techniques were in their infancy in 1985 and the structure of Rubisco was not highly resolved. Instead, we had a well-characterized mutant that required the development of a hypothesis and the execution of experiments that would test the hypothesis. Essential to success was the support given by the senior member of the team, Bill Ogren, who would often provide encouragement by stating that "no important biological process is regulated spontaneously.' In addition, Bill Ogren frequently played the 'devil's advocate,' providing much needed critical evaluation of the experiments to balance our youthful (Figure 1) exuberance. The identification of activase is a testament to the power of a mutational approach in investigations of photosynthesis, not only for confirming the known, but also for revealing unknown aspects of the process.

\section{Acknowledgments}

We thank the many graduate students, postdocs and visitors who have worked in our labs over the years, but especially Anna Brooks, Chris Chastain, Bill Campbell, Mark Chatfield, Ross Lilley, Keith Roesler, Kit Steusand, Jeff Werneke, and Ray Zielinski, who also made important contributions to developing the activase story in the 1980s.

\section{Note}

${ }^{1}$ The notion that Rubisco regulation is a spontaneous process controlled by light-driven changes in stromal $\mathrm{pH}$ and $\mathrm{Mg}^{2+}$ (Lorimer et al. 1976; Heldt et al. 1978) persists today in some textbooks despite conclusive evidence to the contrary.

\section{References}

Bahr JT and Jensen RG (1974) Ribulose diphosphate carboxylase from freshly ruptured spinach chloroplasts having an in vivo $\mathrm{Km}$ $\left[\mathrm{CO}_{2}\right]$. Plant Physiol 53: 39-44

Berry JA, Lorimer GH, Pierce J, Seemann JR, Meek J and Freas S (1987) Isolation, identification, and synthesis of 2carboxyrarbinitol 1-phosphate, a diurnal regulator of ribulosebisphosphate carboxylase activity. Proc Natl Acad Sci USA 84: 734-738

Bowes G, Ogren WL and Hageman RH (1971) Phosphoglycolate production catalyzed by ribulose diphosphate carboxylase. Biochem Biophys Res Commun 45: 716-722

Duff AP, Andrews TJ and Curmi PMG (2000) The transition between the open and closed states of Rubisco is triggered by the inter-phosphate distance of the bound bisphosphate. J Mol Biol 298: 903-916
Gutteridge S, Parry MAJ, Burton S, Keys AJ, Mudd A, Feeney J, Servaites JC and Pierce J (1986) A nocturnal inhibitor of carboxylation in leaves. Nature 324: 274-276

Heldt HW, Chon CJ and Lorimer GH (1978) Phosphate requirement for the light activation of ribulose-1,5-bisphosphate carboxylase in intact spinach chloroplasts. FEBS Lett 92: 234-240

Holbrook GP, Bowes G and Salvucci ME (1989) Degradation of 2-carboxyarabinitol 1-phosphate by a specific chloroplast phosphatase. Plant Physiol 90: 673-678

Holtum JAM and Latzko E (1987) Report, Seventh International Congress on Photosynthesis and Carbon Metabolism. Photosynthetica 21: 391-392

Jordan DB and Chollet R (1983) Inhibition of ribulose bisphosphate carboxylase by substrate ribulose 1,5-bisphosphate. J Biol Chem 258: $13752-13758$

Kawashima N and Wildman SG (1970) Fraction I protein. Annu Rev Plant Physiol 21: 325-358

Laing WA and Christeller JT (1976) A model for the kinetics of activation and catalysis of ribulose 1,5-bisphosphate carboxylase. Biochem J 159: 563-570

Lendzian KJ (1978) Activation of ribulose-1,5-bisphosphate carboxylase by chloroplast metabolites in a reconstituted spinach chloroplast system. Planta 143: 291-296

Lorimer GH (1979) Evidence for the existence of discrete activator and substrate sites for $\mathrm{CO}_{2}$ on ribulose-1,5-bisphosphate carboxylase. J Biol Chem 254: 5599-5601

Lorimer GH, Badger MR and Andrews TJ (1976) The activation of ribulose-1,5-bisphosphate carboxylase by carbon dioxide and magnesium ions: equilibria, kinetics, a suggested mechanism, and physiological implications. Biochemistry 15: 529-536

Machler F and Nosberger J (1980) Regulation of ribulose bisphosphate carboxylase activity in intact wheat leaves by light, $\mathrm{CO}_{2}$, and temperature. J Exp Bot 31: 1485-1491

Perchorowicz JT and Jensen RG (1983) Photosynthesis and activation of ribulose bisphosphate carboxylase in wheat seedlings. Plant Physiol 71: 955-960

Perchorowicz JT, Raynes DA and Jensen RG (1981) Light limitation of photosynthesis and activation of ribulose bisphosphate carboxylase in wheat seedlings. Proc Natl Acad Sci USA 78: 2985-2989

Pon NG, Rabin BR and Calvin M (1963) Mechanism of the carboxydismutase reaction. I. The effect of preliminary incubation of substrates, metal ion and enzyme on activity. Biochem Zeit 338: 7-19

Purich DL (2001) Enzyme catalysis: a new definition accounting for noncovalent substrate- and product-like states. Trends Biochem Sci 26: 417-421

Robinson SP and Portis AR Jr (1988) Release of the nocturnal inhibitor, carboxyarabinitol-1-phosphate from ribulose bisphosphate carboxylase/oxygenase by Rubisco activase. FEBS Lett 233: 413-416

Robinson SP and Portis AR Jr (1989) Adenosine triphosphate hydrolysis by purified Rubisco activase. Arch Biochem Biophys 268: 93-99

Salvucci ME, Portis AR Jr and Ogren WL (1985) A soluble chloroplast protein catalyzes ribulosebisphosphate carboxylase/oxygenase activation in vivo. Photosynth Res 7: 193-201

Salvucci ME, Holbrook GP, Anderson JC and Bowes G (1988) NADPH-dependent metabolism of the ribulose bisphosphate carboxylase-oxygenase inhibitor 2-carboxyarabinitol 1phosphate by a chloroplast protein. FEBS Lett 231: 197-201

Schreuder HA, Knight S, Curmi PMG, Andersson I, Cascio D, Branden C-I and Eisenberg D (1993) Formation of the active 
site of ribulose-1,5-bisphosphate carboxylase/oxygenase by a disorder-order transition from the unactivated to the activated form. Proc Natl Acad Sci USA 90: 9968-9972

Seemann JR, Kobza J and Moore BD (1990) Metabolism of 2 carboxyarabinitol 1-phosphate and regulation of ribulose-1 5bisphosphate carboxylase activity. Photosynth Res 23: 119-130

Sicher RC, Hatch AL, Stumpf DK and Jensen RG (1981) Ribulose 1,5-bisphosphate and activation of the carboxylase in the chloroplast. Plant Physiol 68: 252-255

Somerville CR, Portis AR Jr and Ogren WL (1982) A mutant of Arabidopsis thaliana which lacks activation of RuBP carboxylase in vivo. Plant Physiol 70: 381-387

Streusand VJ and Portis AR Jr (1987) Rubisco activase mediates ATP-dependent activation of ribulose bisphosphate carboxylase. Plant Physiol 85: 152-154
Walker DA (1973) Photosynthetic induction phenomena and the light activation of ribulose diphosphate carboxylase. New Phytol 72: 209-235

Weis E (1981a) Reversible heat-inactivation of the Calvin cycle: a possible mechanism of the temperature regulation of photosynthesis. Planta 151: 33-39

Weis E (1981b) The temperature-sensitivity of dark-inactivation and light-activation of the ribulose-1,5-bisphosphate carboxylase in spinach chloroplasts. FEBS Lett 129: 197-200

Weissbach A and Horecker BL (1989) The path of carbon in photosynthesis - back to the future. Plant Mol Biol Report 7: 159-169

Wildman SG and Bonner J (1947) The proteins of green leaves. I. Isolation, enzymatic properties and auxin content of spinach cytoplasmic proteins. Arch Biochem 14: 381-413 